\title{
The Practical Research on Signal Processing System of Electronic Information Engineering
}

\author{
Shaozhong $\mathrm{Hu}^{1, \text { a }}$ \\ ${ }^{1}$ Xinyu University, Xinyu, Jiangxi, 338000 \\ a email
}

Keywords: Signal Processing System, Electronic Information Engineering, Practical Research

\begin{abstract}
With the continuous development of information technology and computer technology, the development of digital signal processing technology has appeared in people's sight and has been paid more and more attention. Nowadays, it has become an important technical subject. The emergence of DSP makes digital signal processing technology more in-depth development, but also makes the signal processing technology develop towards a more extensive area, such as medical electronics, computer, military, communications, consumer electronics and other fields. The comprehensive practice of electronic information engineering is a typical application practice, which has far-reaching significance.
\end{abstract}

\section{Introduction}

With the development of information technology revolution and the rapid development of computer technology, digital signal processing technology has gradually developed into a very important technical discipline. Integrated digital signal processor DSP (Digital Signal Processor) the emergence of a variety of digital signal processing algorithms provide the possibility. This has greatly promoted the further development of digital signal processing technology; on the other hand, digital signal processing applications continue to expand. Today, DSP has been widely used in general digital signal processing, communications, control, instrumentation, medical electronics, consumer electronics, computers, military and other fields. With the continuous improvement of DSP device performance, DSP signal processing, especially real-time signal processing has become a new hotspot of today and future technology development. Application of DSP to "Electronic Information Engineering Integrated Practice" is a typical application of DSP in the field of education and scientific research. It is of great significance to improve the teaching of electronic information engineering practice.

\section{The Advantages of DSP in the Practice of Electronic Information Engineering}

With the DSP signal processing system design, you can design a variety of software to perform a variety of signal processing tasks. For example, to the DSP load data acquisition related procedures make it a data acquisition processor, and to the DSP load modulation, demodulation related procedures it has become a modem processor. Another example, a digital filter can be programmed to complete the low-pass, high-pass, band-pass, with the resistance of different filtering tasks, without the need to change the hardware. DSP for signal processing system of this programmability, providing a variety of signal processing algorithms for flexible application of the signal processing algorithm also has important significance.

Unlike ordinary microprocessors, DSP to abandon von Neumann structure, the use of Harvard architecture, the program and data storage space separately, each with its own address bus and data bus. This allows processing instructions and data to be processed at the same time, thus greatly improving the processing efficiency. At the same time, the DSP sets the hardware multiply/accumulator can be completed in a single instruction cycle multiplication/accumulation operation. High-speed data transmission capability is one of the keys of DSP for high-speed real-time processing. Most of the DSP now set up a separate DMA bus and its controller, without affecting the basic or not affect the speed of DSP processing, for parallel data transmission and the 
transfer rate can reach hundreds of megabytes per second.

DSP device is based on ultra-large-scale integrated circuit technology and computer technology developed high-speed high single-chip computer. Its small size, strong function, small power consumption, systematic, easy to use, cost-effective, has been widely used.

\section{DSP Meaning used for the System Platform}

"Electronic Information Engineering Integrated Practice" course and covers a wide range of content, the use of traditional experimental equipment and experimental means to complete these practical content, the process is more complex, more scattered experimental equipment, maneuverability is not good. Some practical courses, especially involves complex digital signal processing, the traditional experimental equipment and means difficult to do. Although some practical procedures are carried out by means of computers, some practices, for example, involve real-time processing of signals, often involving convolution, filtering, fast Fourier transform, Hilbert transform, matrix inversion, etc. High-speed computing practice, computer processing is also facing difficulties, which is an urgent need to improve the practical environment to adapt to new experimental needs.

DSP device high-speed digital signal processing capability and its peripheral signal processing circuit and convenient interface design, making it in signal processing, especially real-time high-speed signal processing more and more widely used. "Electronic Information Engineering Practice" involves many of the signal processing algorithms can be used to achieve DSP, coupled with the programmable DSP, making most of the integrated practice can be completed on a DSP chip. Therefore, the realization of DSP processor and PC computer combination, take full advantage of the computer's powerful operating functions and DSP high-speed signal processing capabilities and programmable features, you can achieve "Electronic Information Engineering Practice" course most of the application requirements, you can To achieve this course of practice systematic, integrated.

\section{Basic Structure of the System}

It is composed of a DSP processing part and a computer operation control part. Real-time channel from the input channel A/D circuit, the output channel D/A circuit. Memory includes read-only memory and static memory. Read-only memory as the program memory, static memory is used as both data storage and program storage. DSP interface with OSP data transmission and control of two functions. The computer part mainly includes the labor SA interface, the practice contact surface, the support software, the processing center several parts. Work SA interface has two aspects of computer data transmission and control functions. Practical interface for the man-machine dialogue window, it is both the entrance and the terminal. The support software is the DS application package that is placed on the computer and determines the application functionality to be completed by the system. The computer processing center coordinates processing data transmission, control, reprocessing and so on.

(1) Pre-amplifier: the input analogs signal voltage amplification, so that the output level can meet the $\mathrm{A} / \mathrm{D}$ requirements.

(2) Analog low-pass filter: In order to meet the Nyquist sampling theorem, so that real-time signal sampling can be restored without distortion, the highest signal spectrum must be less than half the sampling frequency. Thereby design the cutoff frequency of the low-pass filter.

(3) Channel selector switch 1: To suit the needs of the experiment, set the selector switch. Throughput, the input port can be connected to signal generators and other special equipment to meet the requirements of a variety of experiments.

(4) A/D converter: the input port sent to the analog signal into a digital signal, sent to the DSP for digital signal processing. The A/D sampling frequency can be programmed by the DSP control to meet different processing requirements.

(5) D/A converter: the data processing results into analog signals.

(6) Analog low-pass filter: filter D/A transformation of the high-frequency components. 
(7) Power amplifier: the D/A converted analog amplified to the level suitable for observation instruments.

(8) Channel selector switch 2: Set to allow the experimenter to recognize the characteristics of the D/A device. You can observe the D/A device directly output signal.

(9) OSP chip: the core processor. The completion of a variety of digital signal processing algorithms and to achieve data input and output access and port control.

(10) Program memory: Loads the DSP handler. Part of the curing in the EPROM is in the DSP initialization and communication procedures and the other part of the PC down to the RAM in a variety of DSP applications.

(11) Data memory: DSP processing input and output data storage. Consider the processing board to adapt to the needs of computer simulation of DSP processing data and set a large data memory is more conducive to analog data directly loaded into the DSP external memory, which will help improve processing speed. In addition, the large data memory is also some real-time processing algorithm itself is required.

(12) High-speed data transmission channel: the main task is to transfer data between the DSP and PC fast. The processing here is a parallel port mode of operation.

(13) Command and control interface: Complete DSP and PC communication handshake, and PC-to-DSP operation control. Mainly to send control words to the DSP and DSP interrupt signal sent two aspects.

(14) Serial communication expansion interface: In order to adapt to other communication requirements, the DSP's serial port and some control port out, so that in the future on the basis of the system function expansion. Such as network-related function and so on.

ISA communication interface unit: the realization of DSP data and PC data between the interaction, and control signal transmission. ISA on the 10-port communication are used to visit the way, do not use the memory mapping. PC processing unit: to complete the DSP data further processing, and as the main controller of the various operations. Experimental interface: to achieve human-computer interaction. On the one hand, the experimental interface to complete the DSP processing results display, data analysis and preservation and other treatment. On the other hand, it is a functional control region in which various experimental operations are performed by the experimenter. Through it can operate the DSP processing board hardware, according to the experimental requirements to set the relevant parameters, you can also control the experimental interface itself, such as display ratio, dynamic, static and so on. Support Software: These are the various DSP application packages. You can easily through the PC interface on the experimental operation to achieve the required DSP application load, so that the completion of the corresponding processing functions. Put DSP applications on the computer instead of the DSP application programming board on the ROM in the general practice, eliminating the DSP processing board on the use of large-capacity ROM, but also greatly increased the flexibility to DSP applications Development is not limited to ROM hardware, and is not limited by development time. The DSP application on the PC microcomputer can be modified at any time, or any further software development may be required at any time, without the need to manipulate the processing board hardware. This way to achieve the system's multi-function.

\section{Application of Data Signal Processing Technology in Integrated Practice of Electronic Information Engineering}

The comprehensive practice of electronic information engineering involves a wide range of content, if using the traditional experimental methods and experimental equipment to practice it will be very complicated. In addition, the arrangement of the experimental apparatus is relatively dispersed, and thus, has a comparatively poor operability. If you are dealing with more complex signals, the use of traditional methods and equipment, it is difficult to get accurate data. Although the traditional experimental methods in some of the practical aspects of the application to the computer, but encountered in the high-speed operation of the signal, the computer is no way to be competent. Digital signal processing technology has a powerful signal processing capabilities and more 
convenient peripheral signal interface. Thus it will be popular in the market. In the integrated practice of electronic information engineering, a lot of signal processing algorithms can be realized by digital signal processing technology. It can be said that digital signal processing technology for electronic information engineering integrated practice provides a relatively new development ideas.

Digital signal processing system unit is mainly used for digital signal processing and the task is to complete most of the signal processing. The upper part of the figure is the signal input and output channels, the lower part of the computer microcomputer and digital signal processing system unit connection. In the whole digital signal processing system, the computer microcomputer occupies the dominant position, the digital signal processing system unit belongs to the subordinate position. The computer to digital signal processing system unit is for effective control, digital signal processing system to complete data control, processing and transmission tasks.

\section{Conclusion}

Electronic information engineering is a relatively complex system and the traditional experimental method is difficult to deal with the practice of the process. And thus we need the more simple and convenient means to operate it. Along with the development of digital information processing technology, the high-speed operation of the information carried out an effective treatment. And thus promote the comprehensive implementation of electronic information engineering.

\section{References}

[1] Zhao Jie. DSP in the integrated application of electronic information engineering analysis [J]. Information Engineering (electronic production), 2008 (8)

[2] Jia Liang, Bai Richang, Zhang Jing. Study on interactive whiteboard system based on interactive technology [J]. Digital Technology and Applications, 2014 (8): 11

[3] Deng Kaiyong. DSP-based signal processing systems in electronic information [D]. University of Electronic Science and Technology, 2002

[4] Ma Lin. Automation Technology, Computer Technology [J]. Chinese Journal of Radio Electronics, 2010 (04): 167-247.

[5] Zhou Zhiao. Communication [J]. Chinese Journal of Radio Electronics, 2011 (02): 92-130. 